\title{
Dietary Strategies of Elite Natural Bodybuilders
}

\author{
A.J. Chappell and T. Simper \\ Food and Nutrition Group, Sheffield Business School, Sheffield Hallam University S1 1WB, UK.
}

Competitive bodybuilders employ a combination of resistance training, cardiovascular $(\mathrm{CV})$ exercise, calorie reduction, supplementation regimes and peaking strategies in order to lose fat mass and maintain fat free mass ${ }^{(1)}$. Although recommendations exist for contest preparation, applied research is limited ${ }^{(2,3)}$ and data on the contest preparation regimes of bodybuilders often involve small cohorts ${ }^{(4)}$.

The British Natural Bodybuilding Federation (BNBF) runs an annual national competition for elite bodybuilders. Competitors are subject to stringent drug testing and have to undergo a polygraph prior to taking part in this event. Study of this cohort provides an opportunity to examine the dietary and training practices of elite natural bodybuilders. Here we report the results of a recent crosssectional study of bodybuilders competing at the BNBF finals.

Fifty-one competitors (35 male and 16 female) completed a 34-item questionnaire, which assessed dietary and training habits at three time points throughout contest preparation. At each time point participants recorded food intake over a 24-hr period in both grams and portions. Competitors were split into two groups depending on their contest placing. A "Placed" competitor finished in the top 5 of their category, and a "Non -placed (DNP) competitor finished outside of the top 5. Nutrient analysis was performed using Nutritics (version 2.267) and statistical analysis used a repeated measures ANOVA (IBM SPSS (version 24), where statistical significance was determined at $\mathrm{P}<0 \cdot 05$."

The mean preparation time for a competitor was 22 weeks \pm 9 . Nutrient intake of competitors is presented in table 1 . Total carbohydrate $(\mathrm{CHO})$ and fat intake decreased over time in both male and female cohorts $(\mathrm{P}<0.05)$. Moreover, in men protein $(\mathrm{PRO})$ energy increased over the preparation period $(\mathrm{P}=0.04)$, (Start 33\%, Middle $35 \%$, End $38 \%$ ). The amount of $\mathrm{CV}$ exercise performed in addition to resistance training increased in the male but not the female cohort $(\mathrm{P}=0 \cdot 001)$, (Start 18.5 $\pm 19 \cdot 7$ Middle 27.6 $\pm 23 \cdot 2$ End $34 \cdot 1 \pm 25 \cdot 4)$.

Table 1. Macronutrient Intakes of Competitive Natural Bodybuilders during Contest Preparation at Different Stages of their Diet.

\begin{tabular}{|c|c|c|c|c|c|c|c|c|c|c|c|c|c|c|c|c|c|c|}
\hline & \multicolumn{6}{|c|}{ PRO g/kg bw per day } & \multicolumn{6}{|c|}{$\mathrm{CHO} \mathrm{g} / \mathrm{kg}$ bw per day } & \multicolumn{6}{|c|}{ Fat g/kg bw per day } \\
\hline & \multicolumn{2}{|c|}{ Start } & \multicolumn{2}{|c|}{ Middle } & \multicolumn{2}{|c|}{ End } & \multicolumn{2}{|c|}{ Start } & \multicolumn{2}{|c|}{ Middle } & \multicolumn{2}{|c|}{ End } & \multicolumn{2}{|c|}{ Start } & \multicolumn{2}{|c|}{ Middle } & \multicolumn{2}{|c|}{ End } \\
\hline & $\overline{\mathrm{M}}$ & $\mathrm{SD}$ & $\bar{M}$ & SD & $\bar{M}$ & $\mathrm{SD}$ & $\overline{\mathrm{M}}$ & $\mathrm{SD}$ & $\bar{M}$ & $\mathrm{SD}$ & $\bar{M}$ & SD & $\bar{M}$ & SD & $\bar{M}$ & SD & $\bar{M}$ & $\mathrm{SD}$ \\
\hline$ð$ Place & 3.0 & $1 \cdot 0$ & $3 \cdot 1$ & $0 \cdot 9$ & $3 \cdot 3$ & $0 \cdot 9$ & $5 \cdot 1$ & 1.9 & 4.9 & 1.8 & $4 \cdot 6$ & $2 \cdot 2$ & $0 \cdot 8$ & $0 \cdot 3$ & $0 \cdot 8$ & $0 \cdot 3$ & $0 \cdot 9$ & $1 \cdot 2$ \\
\hline$ð \mathbf{N P}$ & $2 \cdot 7$ & 0.6 & $2 \cdot 8$ & $0 \cdot 6$ & $2 \cdot 7$ & $0 \cdot 8$ & 3.7 & $0 \cdot 9$ & $3 \cdot 6$ & 1.6 & $3 \cdot 6$ & $2 \cdot 7$ & $0 \cdot 9$ & $0 \cdot 6$ & $0 \cdot 9$ & $0 \cdot 8$ & $0 \cdot 6$ & $0 \cdot 3$ \\
\hline ㅇ Place & $2 \cdot 7$ & 0.6 & $2 \cdot 9$ & $0 \cdot 5$ & $2 \cdot 9$ & $1 \cdot 0$ & $3 \cdot 8$ & $2 \cdot 2$ & $3 \cdot 7$ & $2 \cdot 0$ & $3 \cdot 5$ & 1.6 & 0.7 & $0 \cdot 3$ & 0.7 & $0 \cdot 3$ & 0.5 & $0 \cdot 1$ \\
\hline 우 NP & $2 \cdot 7$ & 0.5 & $2 \cdot 7$ & $0 \cdot 5$ & $2 \cdot 9$ & 0.6 & $4 \cdot 0$ & 0.7 & $3 \cdot 5$ & 1.0 & $3 \cdot 1$ & 1.5 & $0 \cdot 9$ & 0.4 & $0 \cdot 8$ & $0 \cdot 3$ & 0.8 & 0.4 \\
\hline
\end{tabular}

Abbreviations, PRO, Protein, CHO, Carbohydrate, ơ Male, o Female $M$, mean, g/kg bw per day, grams per kilogram of bodyweight per day, Placed, Competitors who finished in the top 5, NP, competitors who finish out of the top 5.

In conclusion, the mean intake of natural bodybuilders reflected a high $\mathrm{PRO}$, high $\mathrm{CHO}$, low fat diet. As preparation progressed, the amount of $\mathrm{CV}$ exercise performed increased, while total fat and carbohydrates intakes decreased. Protein intake was maintained at a high level presumably in an attempt to preserve muscle tissue.

1. Robinson SL, Lambeth-Mansell A, Gillibrand G et al. (2015) JISSN 12, 20.

2. Helms ER, Aragon AA \& Fitschen PJ (2014) JISSN 12, 20.

3. Helms ER, Fitschen PJ, Aragon AA et al. (2015) J Sports Med Phys Fit 55, 164-178.

4. Spendlove J, Mitchell L, Gifford J et al. (2015) Sports Med 45, 1041-1063. 